\title{
Diagnóstico de nutrimento compuesto e interacciones nutrimentales en chile Jalapeño (Capsicum annuum L.) en suelos semiáridos
}

\section{Compositional nutrients diagnosis and nutrimental interactions in Jalapeño pepper (Capsicum annuum L.) in semiarids soils}

\author{
José Apolinar Ramírez-Ibarra ${ }^{1}$, Enrique Troyo-Dieguez ${ }^{2}$, Pablo Preciado-Rangel ${ }^{3}$, Manuel Fortis-Hernández ${ }^{3}$, \\ Miguel Ángel Gallegos-Robles ${ }^{4}$, Cirilo Vázquez-Vázquez ${ }^{4}$, Juan Luis Ríos-Plaza ${ }^{4}$, José Luis \\ García-Hernández ${ }^{4 *}$ \\ ${ }^{1}$ Doctorado Institucional en Ciencias Agropecuarias y Forestales. Universidad Juárez del Estado de Durango. Durango, Dgo., \\ México \\ ${ }^{2}$ Centro de Investigaciones Biológicas del Noroeste, S. C. Mar Bermejo Núm. 195, Col. Playa Palo Santa Rita, CP. 23090, La \\ Paz, Baja California Sur, México \\ ${ }^{3}$ Instituto Tecnológico de Torreón. Carretera Torreón-San Pedro, km 7.5. Ejido Anna, Torreón, Coahuila, México \\ ${ }^{4}$ Facultad de Agricultura y Zootecnia. Universidad Juárez del Estado de Durango. Ej. Venecia, Gómez Palacio, Dgo. México \\ *Corresponding autor: luis_garher@hotmail.com.
}

Artículo científico recibido: 17 de marzo de 2016, aceptado: 09 de noviembre de 2016

RESUMEN. Es fundamental conocer los requerimientos nutricionales específicos de cada genotipo de chile, así como su interacción con los diversos ambientes donde se produce. Los objetivos de este estudio fueron estimar las normas nutrimentales mediante la técnica de diagnóstico de nutrimento compuesto (DNC) de chile jalapeño Autlán en la Comarca Lagunera; y analizar las interacciones nutrimentales entre los nutrimentos y su relación con el rendimiento por medio de correlaciones. Se tomó una muestra de 100 plantas a las que se les determinó el contenido de N, P, K, Ca y $\mathrm{Mg}$, para correlacionarlas con el rendimiento. Se calculó un rendimiento mínimo de referencia de $52.09 \mathrm{t} \mathrm{ha}^{-1}$ y se determinaron los rangos óptimos de estos macro-nutrimentos en el tejido foliar de $\mathrm{N}(4.57 \pm 0.25), \mathrm{P}(0.18 \pm 0.03)$, $\mathrm{K}(6.80 \pm 1.04), \mathrm{Ca}(2.46 \pm 0.17)$ y $\mathrm{Mg}(0.64 \pm 0.07)$ para la obtención de altos rendimientos. El orden decreciente de extracción de nutrimentos en la floración fue $\mathrm{K}>\mathrm{N}>\mathrm{Ca}>\mathrm{Mg}>\mathrm{P}$, patrón similar al de otros cultivares de chile, pero que difiere de otras especies cutivadas como la sábila (Aloe vera L.) o el nopal (Opuntia ficus-indica L.). Se presentaron los sinergismos: $\mathrm{N}$ y $\mathrm{P}, \mathrm{N}$ y K, $\mathrm{P}$ y K, y $\mathrm{Ca}$ y $\mathrm{Mg}$. Mientras que el fósforo fue el único nutrimento que presentó correlación positiva con el rendimiento.

Palabras clave: Rendimiento, correlaciones, normas nutrimentales, concentración.

ABSTRACT. It is fundamental to know nutrimental requierements of every hot pepper genotype, as well as its interaction with the several environments where it is cropped. The aims of this study were to estimate nutrient norms by using the technique of compositional nutrient diagnosis (CND) for hot pepper type jalapeño Autlán in the Comarca Lagunera during the cycle spring-summer 2013 and to analize the nutrimental interactions among the nutrients and its relationship with yield by using the Pearson correlations approach. With this purpose, it was taken a sample 100 plants on which was determined the content of $\mathrm{N}, \mathrm{P}, \mathrm{K}, \mathrm{Ca}$ and $\mathrm{Mg}$ and determinations were correlated to yield. It was calculated a minimum reference yield of $52.09 \mathrm{t} \mathrm{ha}^{-1}$ and optimal ranges of these macro-nutrients on foliar tissue were determined as follow $\mathrm{N}(4.57 \pm 0.25), \mathrm{P}(0.18 \pm 0.03), \mathrm{K}(6.80 \pm 1.04), \mathrm{Ca}(2.46 \pm 0.17)$ y $\mathrm{Mg}(0.64 \pm 0.07)$ for achievement of high yields. Decrecent order of nutrimental extractions on flowering stage were $\mathrm{K}>\mathrm{N}>\mathrm{Ca}>\mathrm{Mg}$ $>\mathrm{P}$, similar pattern of other pepper cultivars, but notabilly different to other cropped species as sábila (Aloe vera L.) or nopal (Opuntia ficus-indica L.). There were observed the folloeing sinergisms N and $\mathrm{P}, \mathrm{N}$ and $\mathrm{K}, \mathrm{P}$ and $\mathrm{K}, \mathrm{Ca}$ and $\mathrm{Mg}$. While phosphorus was the unique nutrient that showed positive correlation to yield.

Key words: Yield, correlations, normas nutrient norms, concentration. 


\section{INTRODUCCIÓN}

El cultivo de chile ( $C$. annuum) pertenece a la familia solanáceas, y se produce en grandes áreas en el mundo (FAOSTAT 2016). Su importancia radica en su valor nutraceútico y su gran popularidad en la alimentación (Vera-Guzmán et al. 2011). Actualmente se reconoce su importancia debido a su contenido de vitamina C (Mateos et al. 2013). Además de sus propiedades terapéuticas como analgésico, anti-inflamatorio y antioxidante (GonzálezZamora et al. 2015). Su alto contenido de vitaminas, principalmente $\mathrm{A}$ y $\mathrm{C}$, su sabor agradable y estimulante, hacen que esté presente en miles de recetas de la cocina de todos los países (Bortolotti 2013). Su popularidad y demanda es creciente a nivel mundial, por lo que actualmente es una de las especies vegetales más usadas en la cocina (Bosland 2010). El fruto de los chiles de la especie C. annuum tiene efectos medicinales contra el cáncer, estimulan el sistema inmunológico, previenen las enfermedades cardiovasculares y retrasan el proceso de envejecimiento, (González-Zamora et al. 2013).

El chile jalapeño es de los de mayor importancia económica, por su amplio consumo, alta rentabilidad y gran demanda de mano de obra (Macías-Duarte et al. 2012). Tomando en cuenta que la población mundial ha crecido a un ritmo muy acelerado, se estima que será de 2400 millones de personas mas en el 2050. Por lo que es necesario optimizar los recursos para cumplir con los requerimientos alimenticios de la población (Delgado et al. 2011).

Se han desarrollado técnicas de diagnóstico nutrimental de las plantas cultivadas (GarcíaHernández et al. 2004). Entre las que se encuentran el valor crítico (VC) (Bates 1971), sistema integrado de diagnóstico y recomendación (SIDR) (Walworth y Sumner 1987) y diagnóstico de nutrimento compuesto (DNC) (Parent y Dafir 1992). En el caso de la técnica de VC, una concentración se define de forma arbitraria, por lo general se considera el 90 ó $95 \%$ del rendimiento máximo y se supone que; excepto el nutrimento de estudio, los demás están en cantidades suficientes y no excesivas (Ware et al. 1982). En los casos de las técnicas SIDR y DNC la subpoblación de altos rendimientos se selecciona de manera diferente. Con respecto al SIDR, no se ha establecido un procedimiento matemático formal para dividir el total de observaciones en dos grupos, uno de alto y otro de bajo rendimiento, sino que se considera el rendimiento promedio regional del cultivo. Esa partición sí se logra con la técnica DNC al considerar la relación cúbica entre el rendimiento y cada una de las funciones de proporción de varianza acumulada (Parent y Dafir 1992). Al respecto Khiari et al. (2001) demostraron que los índices nutrimentales estimados con la técnica DNC se distribuyen de forma simétrica con respecto a un valor nulo de balance nutrimental y se correlacionan mejor con el rendimiento. La técnica DNC se ha utilizado en cultivos anuales (Arroyo-Vargas et al. 2013) y especies perennes (García-Hernández et al. 2006,2009 ). Por lo anterior el objetivo fue estimar las normas nutrimentales mediante la técnica de diagnóstico de nutrimento compuesto para chile jalapeño y analizar las interacciones nutrimentales entre los elementos nutritivos y su relación con el rendimiento.

\section{MATERIALES Y MÉTODOS}

\section{Establecimiento de cultivo y muestreo}

Se estableció en campo en el Ejido Florencia, municipio de Francisco I. Madero, Coahuila, México, en el ciclo primavera-verano 2013. El ejido, se ubica en las coordenadas $25^{\circ} 50^{\prime} 22.32^{\prime} \mathrm{LN}$ y $103^{\circ} 18^{\prime}$ 59.11" LO, a una altitud de 1107 msnm. La temperatura media anual es de $22.1{ }^{\circ} \mathrm{C}$, la precipitación pluvial de $258 \mathrm{~mm}$ y la evaporación anual media es 10 veces mayor. Los suelos son de aluvión, profundos y arcillosos (García-Hernández et al. 2009).

Antes de la siembra se obtuvieron 10 muestras de suelo, de la capa de $0-30 \mathrm{~cm}$ del sitio para determinar las características físicas y químicas. El suelo es migajón arenoso, alcalino con $\mathrm{pH}$ de 8.4, baja salinidad con CE de $1.46 \mathrm{dS} \mathrm{m}^{-1}$, el contenido de $\mathrm{MO}$ es de $1.1 \%, 17 \mathrm{mg} \mathrm{kg}^{-1}$ de $\mathrm{N}, 14 \mathrm{mg} \mathrm{kg}^{-1}$ de $P, 1.30$ meq $L^{-1}$ de $K, 5.4$ meq $L^{-1}$ de Ca y 6.8 meq $\mathrm{L}^{-1}$ de $\mathrm{Mg}$. 


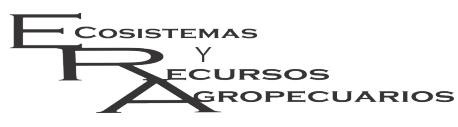

Se estableció el cultivar de chile jalapeño cv. Autlán, en un arreglo espacial en bordos de doble hilera, acolchado plástico negro de $1.2 \mathrm{~m}$ de ancho, calibre de 100 micras de grosor con riego por goteo. La distancia entre bordos fue de $1.80 \mathrm{~m}$ y entre planta de $30 \mathrm{~cm}$, para obtener una densidad de 37000 plantas ha ${ }^{-1}$. La nutrición se realizó con fertirriego, aplicando 120 y $60 \mathrm{~kg} \mathrm{ha}^{-1}$ de $\mathrm{N}$ y $\mathrm{P}$, respectivamente; usando como fuente de fertilización fosfato monoamónico y sulfato de amonio.

Las plagas y enfermedades que se presentaron fueron el picudo del chile (Anthonomus eugenii Cano) y cenicilla polvorienta (Oidiopsis taurica Salmon), las cuales se controlaron con los productos orgánicos Insecta Plus y PHC - Milstop Plus ${ }^{\circledR}$, ambos productos certificados por OMRI (Organic Materials Review Institute) para uso en la producción orgánica. Del total de plantas establecidas, durante la etapa de floración se tomó una muestra de 100 unidades de dos plantas por unidad de muestreo para el cálculo de las normas. Se tomaron siete hojas del tercio medio de cada planta muestreada (García- Hernández et al. 2004), más otras siete de la planta contigua hacia el norte, para tener 14 hojas por unidad de muestreo. Cada muestra foliar compuesta de 14 hojas se limpió con agua destilada, para luego secarla hasta peso constante en una estufa Marca Shel-lab, modelo FX-5 a $65^{\circ} \mathrm{C}$ durante $24 \mathrm{~h}$, para luego molerlas y guardarlas en bolsas de plástico para su uso en la determinación del porcentaje total de N, P, K, Ca y Mg. De las mismas plantas muestreadas se obtuvo el rendimiento total de fruto fresco de cada unidad de muestreo y se extrapoló a t ha ${ }^{-1}$. El rendimiento total se relacionó con las concentraciones de los nutrimentos para estimar las normas nutrimentales, el cual se obtuvo de la suma de los rendimientos parciales de cinco cortes, que se realizaron a los 131, 138, 145, 152 y 159 d después del trasplante. Los análisis químicos realizados a las muestras se llevaron a cabo en los laboratorios del Centro de Investigaciones Biológicas del Noroeste (CIBNOR) en La Paz, Baja California Sur. EI N total se determinó por el método de Dummas. El fósforo por colorimetría con el complejo de azul de
Ramirez-Ibarra et al. Normas nutrimentales en chile jalapeño Ecosist. Recur. Agropec. 4(11):233-242,2017

fosfo-molibdato (AOAC 2005), mientras que el $\mathrm{K}$, $\mathrm{Ca}$ y $\mathrm{Mg}$ se estimaron por espectrofotometría de absorción atómica, después de la digestión con $\mathrm{HNO}_{3}$ (Hanton 1992).

\section{Estimación de las normas preliminares}

Las normas nutrimentales DNC se estimaron a partir del procedimiento teórico-matemático descrito por Khiari et al. (2001), García-Hernández et al. (2006), (2009) y Arroyo-Vargas et al. (2013). Para lo cual las concentraciones de los nutrimentos se transformaron a proporciones invariantes (centradas) al dividirlas por la media geométrica. Posteriormente, se determinaron los logaritmos de las proporciones centradas $V_{N}, V_{P}, V_{K}, V_{C a}, V_{M g}, \ldots, V_{R d}$. Para luego calcular las proporciones de varianza $\left[f_{i}\left(V_{x}\right)\right]$ entre grupos de observación asociados a altos y bajos rendimientos, considerando el procedimiento de Cate-Nelson (Nelson y Anderson 1977). Las dos observaciones con mayor rendimiento formaron el grupo de alto rendimiento y el resto de observaciones el grupo de bajo rendimiento; en la siguiente repetición del proceso (iteración) el grupo de alto rendimiento se formó por tres observaciones. Mientras que el de bajo rendimiento por una observación menos que el grupo de bajo rendimiento asociado a la iteración inicial; y así de forma sucesiva. Después, el valor de la función de proporción de varianza acumulada $\left[f_{i}^{c}\left(V_{x}\right)\right]$ se estimó, para cada repetición o paso del proceso, como la suma de las proporciones de varianza. La función de proporción de varianza acumulada se calculó al considerar la proporción entre la sumatoria de las proporciones de varianza en cada iteración y la sumatoria de las proporciones de varianza de todas las iteraciones (constante); donde resulta una función cúbica útil para comparar la fuerza discriminatoria de los logaritmos de las proporciones centradas $\left(V_{x}\right)$ entre los grupos de alto y bajo rendimiento, sobre una escala común. Los puntos de inflexión para diferenciar entre las subpoblaciones de altos y bajos rendimientos se determinaron después de examinar las seis funciones cúbicas ajustadas al considerar las relaciones entre cada función de varianza acumulada 
Ramírez-Ibarra et al.

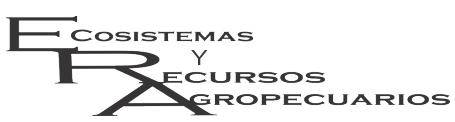

Normas nutrimentales en chile jalapeño

Ecosist. Recur. Agropec.

4(11):233-242,2017

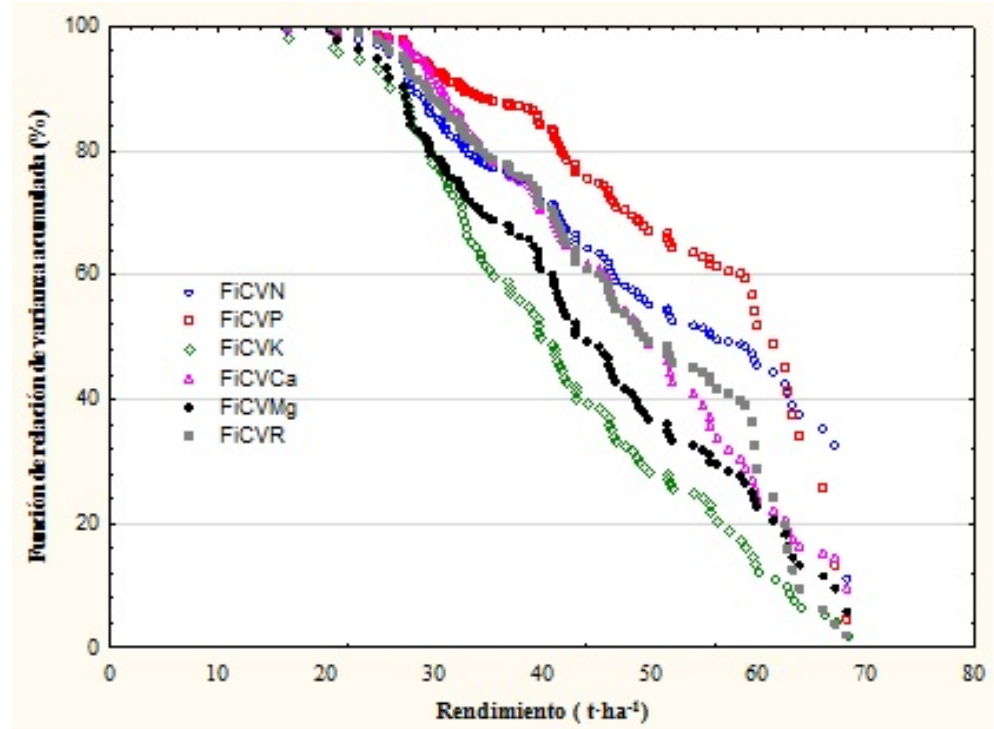

Figura 1. Relación entre el rendimiento de chile jalapeño Autlán y la función de varianza acumulada para estimar el punto de inflexión.

$\left[F_{i(V N)}^{c}, F_{i(V P)}^{c}, \ldots, F_{i(V R)}^{c}\right]$ y el rendimiento.

\section{Interacciones nutrimentales}

Para la identificación de las interacciones entre los nutrimentos analizados en el tejido vegetal y su relación con el rendimiento del cultivo, se estimaron coeficientes de correlación de Pearson (Naiman et al. 1987). Estos coeficientes varían de -1 a +1 , por lo que valores positivos significativos sugieren relaciones sinérgicas, mientras que valores negativos significativos implican relaciones inversas o antagonismos; cuando se analiza la relación entre pares de nutrimentos. Mientras que cuando se relaciona algún nutrimento con el rendimiento, es posible evidenciar dependencia de éste con respecto al nutrimento del caso (Blanco-Macías et al. 2006). Para la generación de las normas preliminares se utilizó el programa Microsoft Excel 2010, mientras que para el cálculo de los coeficientes de Pearson se usó el programa Statistica versión 9.1.

\section{RESULTADOS}

\section{Normas de diagnóstico de nutrimento}

La media aritmética del contenido de $\mathrm{N}, \mathrm{P}$,
$\mathrm{K}, \mathrm{Ca}$ y $\mathrm{Mg}$, en porcentaje, fueron de 4.57, 0.18, $6.78,2.46$ y $0.66 \%$, respectivamente; mientras que la media de rendimiento fue $40.5 \mathrm{t} \cdot \mathrm{ha}^{-1}$ de fruto fresco de chile. El mayor coeficiente de variación lo tuvo el rendimiento de fruto (31\%), mientras que el coeficiente de las concentraciones nutrimentales fue menor de $20 \%$. El orden decreciente de extracción de nutrimentos al momento de la floración fue $\mathrm{K}>$ $\mathrm{N}>\mathrm{Ca}>\mathrm{Mg}>\mathrm{P}$.

Los seis modelos experimentales entre cada función de proporción de varianza acumulada: $\left[F_{i}^{C}\left(V_{N}\right), F_{i}^{C}\left(V_{p}\right), F_{i}^{C}\left(V_{K}\right), F_{i}^{C}\left(V_{C a}\right), F_{i}^{C}\left(V_{M} g\right)\right.$ como $\left.F_{i}^{C}\left(V_{R}\right)\right]$ y el rendimiento (Figura 1 ), se ajustaron a un modelo cúbico (Tabla 1), mediante el coeficiente de determinación $\left(R^{2}>0.99\right)$. Los valores de rendimiento asociados a los puntos de inflexión fueron 31.33, 32.21, 34.67, 52.09, 42.42 y $37.5 \mathrm{t} \mathrm{ha}^{-1}$ para $\mathrm{N}, \mathrm{P}, \mathrm{K}, \mathrm{Ca}, \mathrm{Mg}$ y $\mathrm{R}$, respectivamente (Tabla 1).

El valor crítico que se tomó como rendimiento de referencia fue $52.09 \mathrm{t} \mathrm{ha}^{-1}$, asociado al punto de inflexión del modelo cúbico ajustado al $\mathrm{Ca}$. En la Tabla 2 se muestran las normas preliminares de DNC como medias y desviaciones estándar $\left(V_{x}^{*}\right)$ y DE $V_{x}^{*}$ respectivamente para el cultivar de chile 
Tabla 1.Rendimiento de chile jalapeño cv. Autlán en los puntos de inflexión de las funciónes de varianza acumulada de la proporción de logaritmos centrados en la población estudiada $(n=100)$.

\begin{tabular}{clcc}
\hline Nutrimento & $F(V x)=a Y^{3}+b Y^{2}+c Y+d$ & $\mathrm{R}^{2}$ & Rendimiento t ha $^{-1}$ \\
\hline $\mathrm{N}$ & $y=-0.00005 x^{3}+0.0047 x^{2}-1.5773 x+130.22$ & 0.976 & 31.33 \\
$\mathrm{P}$ & $Y=-0.0008 x^{3}+0.0733 x^{2}-3.01395 x+138.56$ & 0.971 & 32.21 \\
$\mathrm{~K}$ & $y=0.0008 x^{3}-0.0832 x^{2}+0.4197 x+118.13$ & 0.990 & 34.67 \\
$\mathrm{Ca}$ & $y=0.0007 x^{3}-0.1094 x^{2}+2.9799 x+79.307$ & 0.996 & 52.09 \\
$\mathrm{Mg}$ & $y=0.0004 x^{3}-0.0509 x^{2}-0.1034 x+117.86$ & 0.992 & 42.42 \\
$\mathrm{R}$ & $y=-0.00008 x^{3}-0.009 x^{2}-0.7007 x+119.35$ & 0.987 & 37.5 \\
\hline
\end{tabular}

Tabla 2. Normas preliminares de DNC para $d=5$ elementos y rangos de concentración óptimos en chile jalapeño con rendimiento de referencia de $52.09 \mathrm{t} \mathrm{ha}^{-1}$.

\begin{tabular}{lccccc}
\hline Relaciones logarítmicas & Media $V_{x}$ & DE $V_{x}$ & Nutrimento & Media (\%) & DE (\%) \\
\hline $\mathrm{V}^{*} \mathrm{~N}$ & 0.42 & 0.06 & $\mathrm{~N}$ & 4.57 & .25 \\
$\mathrm{~V}^{*} \mathrm{P}$ & -2.83 & 0.17 & $\mathrm{P}$ & 0.18 & 0.03 \\
$\mathrm{~V}^{*} \mathrm{~K}$ & 0.81 & 0.11 & $\mathrm{~K}$ & 6.80 & 1.04 \\
$\mathrm{~V}^{*} \mathrm{Ca}$ & -0.20 & 0.06 & $\mathrm{Ca}$ & 2.46 & 0.17 \\
$\mathrm{~V}^{*} \mathrm{Mg}$ & -1.54 & 0.11 & $\mathrm{Mg}$ & 0.64 & 0.07 \\
$V * R 5$ & 3.35 & 0.07 & $\mathrm{R}$ & 85.34 & 1.24 \\
$\sum V_{x}$ & 0 & - & & & \\
\hline
\end{tabular}

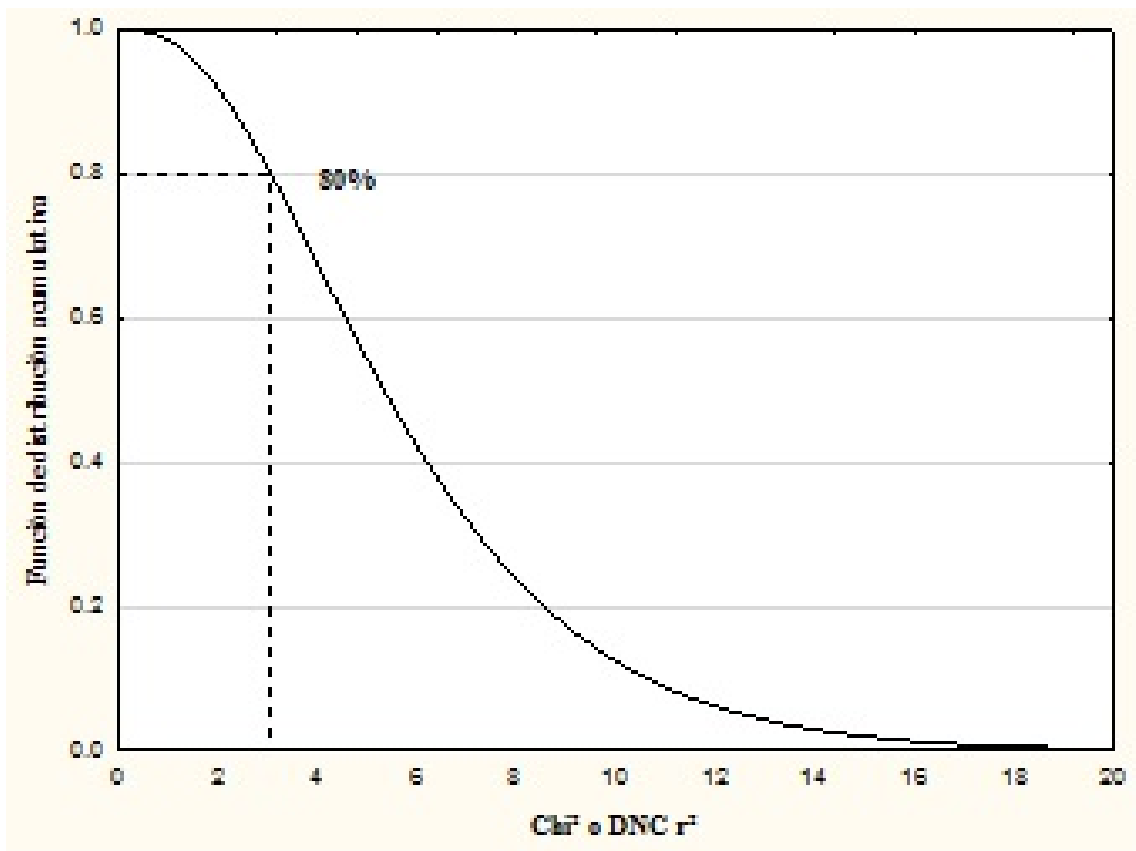

Figura 2. Función de distribución acumulada de $X^{2}$ con seis grados de libertad para obtener el valor crítico teórico de (DNC $r^{2}$ ) en el arreglo $\mathrm{S}^{5}(3.1)$ asociado al $80 \%$ de la población de bajo rendimiento.

jalapeño Autlán. También se muestran los rangos de concentración óptima correspondientes a cada nutrimento. Los índices nutrimentales de DNC IN, $I P, I K, I C a, I M g$, y IRd y de desbalance (DNC, $r^{2}$ ) se obtuvieron de las normas preliminares de DNC y los valores de (DNC $\left.\mathrm{r}^{2}\right)$ tuvieron una distribución de $X^{2}$ con valores $\left(\mathrm{R}^{2}>0.999, \mathrm{p}<0.001\right)$ (Figura 2).

\section{Correlaciones entre nutrimentos y rendimiento} Al calcular la matriz de correlaciones de la población completa se obtiene una idea gen- 
Tabla 3.Concentraciones nutrimentales óptimas para Capsicum, sábila ( $A$. vera) y nopal $(O$. ficus-indica).

\begin{tabular}{lllllr}
\hline Nutrimento & C. annuum & $\begin{array}{l}\text { C. annuum } \\
\text { tipo güero* }\end{array}$ & $\begin{array}{l}\text { C. annuum } \\
\text { 'pimiento' } ¥\end{array}$ & $\begin{array}{l}\text { Sábila } \\
\text { (Aloe vera) } £\end{array}$ & $\begin{array}{r}\text { Nopal (Opuntia } \\
\text { ficus-indica) } €\end{array}$ \\
\hline $\mathrm{N}(\%)$ & $4.0-5.0$ & $4.23-4.83$ & $4.26-4.92$ & $0.75-0.88$ & $0.92-1.03$ \\
$\mathrm{P}(\%)$ & $0.30-0.50$ & $0.30-0.35$ & $0.37-0.46$ & $0.15-0.19$ & $0.30-0.33$ \\
$\mathrm{~K}(\%)$ & $2.50-5.0$ & $3.96-4.46$ & $4.36-5.58$ & $1.94-2.54$ & $3.95-4.99$ \\
$\mathrm{Ca}(\%)$ & $0.90-1.50$ & $2.54-2.88$ & $1.77-2.23$ & $4.25-4.81$ & $4.14-4.60$ \\
$\mathrm{Mg}(\%)$ & $0.30-0.60$ & $0.64-0.71$ & $0.60-0.72$ & $1.05-1.22$ & $1.35-1.59$ \\
\hline
\end{tabular}

Hochmuth (1997), *García-Hernández et al. (2004), ${ }^{¥}$ Arroyo-Vargas et al. (2013), ${ }^{£}$ García-

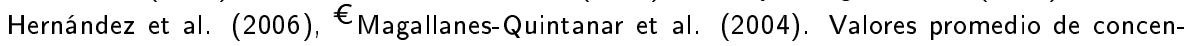
tración \pm 0.5 de la DE.

Tabla 4. Matriz de coeficientes de correlación de Pearson, entre el rendimiento y la concentración de nutrimentos.

\begin{tabular}{lllllll}
\hline & Rendimiento & Nitrógeno & Fósforo & Potasio & Calcio & Magnesio \\
\hline Nitrógeno & 0.038 & & & & & \\
& $\mathrm{p}=0.711$ & & & & & \\
Fósforo & -0.024 & $0.468^{*}$ & & & & \\
& $\mathrm{p}=0.811$ & $\mathrm{p}=0.000$ & & & & \\
Potasio & -0.032 & $0.361^{*}$ & $0.218^{*}$ & & & \\
& $\mathrm{p}=0.749$ & $\mathrm{p}=0.000$ & $\mathrm{p}=0.029$ & & & \\
Calcio & 0.062 & -0.024 & -0.033 & 0.123 & & \\
& $\mathrm{p}=0.542$ & $\mathrm{p}=0.815$ & $\mathrm{p}=0.743$ & $\mathrm{p}=0.223$ & & \\
Magnesio & -0.152 & -0.099 & -0.192 & 0.067 & $0.417^{*}$ & \\
& $\mathrm{p}=0.130$ & $\mathrm{p}=0.329$ & $\mathrm{p}=0.056$ & $\mathrm{p}=0.509$ & $\mathrm{p}=0.000$ & \\
$\mathrm{R}$ & 0.014 & $-0.561^{*}$ & $-0.309^{*}$ & $-0.934^{*}$ & $-0.350^{*}$ & -0.190 \\
& $\mathrm{p}=0.893$ & $\mathrm{p}=0.000$ & $\mathrm{p}=0.002$ & $\mathrm{p}=0.000$ & $\mathrm{p}=0.000$ & $\mathrm{p}=0.058$ \\
\hline
\end{tabular}

* significativo a $\mathrm{p}<0.05$.

eral del grado en que el rendimiento depende de los nutrimentos (Tabla 4). Esta matriz muestra correlaciones significativas $(\mathrm{p} \leq 0.05)$ entre los nutrimentos, se observa que el rendimiento no depende de ninguno de los nutrimentos. Mientras que las correlaciones nutrimentales significativas entre $\mathrm{N}$ y $P(r=0.468), N$ y K $(r=0.361)$, $P$ y $K(r=$ $0.218)$ y, Ca y $\mathrm{Mg}(r=0.417)$ fueron positivas, lo que significa que cuando uno aumenta, el otro también incrementa.

Ninguno de los nutrimentos presentó correlación significativa con el rendimiento. Este resultado no es común en este tipo de estudios, por lo cual se realizaron dos matrices más de coeficientes de correlación de Pearson, entre el rendimiento y los nutrimentos; una al considerar la subpoblación de altos rendimientos y otra la de bajos rendimientos. La matriz de correlaciones de la subpoblación de altos rendimientos mostró que el rendimiento depende de forma significativa $(p \leq 0.05)$ del $P(r=$ $0.494)$, con correlación positiva entre $\mathrm{Ca}$ y $\mathrm{Mg}(\mathrm{r}$
$=0.483$. Mientras que, para la subpoblación de bajos rendimientos se presentó correlación positiva $(p<0.05)$ entre $N$ y $P(r=0.505), N$ y K $(r=$ $0.355), P$ y K $(r=0.222)$ y, Ca y $\mathrm{Mg}(r=0.416)$. $\mathrm{Al}$ analizar las tres matrices de correlaciones, se dedujo que en las matrices de la base de datos completa y en la de bajos rendimientos, ninguno de los elementos nutritivos se correlacionó de forma significativa con el rendimiento. Mientras que en la matriz de altos rendimientos, se observa una correlación positiva y significativa entre el $\mathrm{P}$ y el rendimiento.

\section{DISCUSIÓN}

\section{Normas de diagnóstico de nutrimento com- puesto}

La teoría del método de DNC indica que es recomendable considerar el rendimiento mayor como referencia, para separar las observaciones en dos subpoblaciones, una de alto y otra de bajo 
rendimiento (Parent y Dafir 1992). Al tomar como referencia el valor crítico de rendimiento de 52.09 $\mathrm{t} \cdot \mathrm{ha}^{-1}$, se divide la población completa en dos subpoblaciones; una subpoblación de alto rendimiento, que corresponde al $20 \%$ de las observaciones y la subpoblación de bajo rendimiento que constituye el $80 \%$ de las observaciones. Aunque se presenta una subpoblación de alto rendimiento menor del $32.5 \%$ reportada por Khiari et al. (2001), es posible que sea más confiable, ya que el presente estudio se basa en una población total mayor en un $250 \%$. La tendencia observada coincide con las encontradas por Valdez-Cepeda et al. (2013), ya que al aumentar el número de observaciones en la población total se tiende a disminuir el porcentaje de observaciones de la subpoblación de alto rendimiento.

Los valores encontrados para las normas nutrimentales y las concentraciones óptimas de nutrimentos, sugieren diferencias entre el cultivar de chile jalapeño Autlán (Tabla 2) y otros cultivares del género Capsicum que han sido estudiados por García-Hernández et al. (2004 y 2006); pero también se observan similitudes en el orden decreciente de la extracción de nutrimentos al momento de la floración de $\mathrm{K}>\mathrm{N}>\mathrm{Ca}>\mathrm{Mg}>\mathrm{P}$ para cultivares de chile. Lo más evidente son las diferencias en las concentraciones y la composición (Tabla 3), con lo encontrado en otros tipos de plantas en los que se han calculado estas normas, como sábila ( $A$. vera) (García- Hernández et al. 2006) y nopal (O. ficusindica) (Magallanes-Quintanar et al. 2004). En C. annuum, se reportan concentraciones óptimas de $\mathrm{N}$ (4.0 - 5.0\%), P (0.3 - $0.5 \%), \mathrm{K}(2.5-5.0 \%), \mathrm{Ca}$ (0.9-1.5\%) y Mg (0.3 -0.6\%) (Hochmuth 1997). En el presente trabajo, el $\mathrm{P}$ presentó menor concentración promedio con $0.18 \%$; posiblemente se deba a que los contenidos de $\mathrm{Ca}$ en el suelo son altos (5.4 meq $\mathrm{L}^{-1}$ ) y el $\mathrm{pH}$ alcalino (8.4), por lo que la disponibilidad de $\mathrm{P}$ para los cultivos está limitada por la baja solubilidad del mineral en este $\mathrm{pH}$ del suelo (Johnson et al. 2003). Los nutrimentos K, Ca y $\mathrm{Mg}$ (Tabla 2) tuvieron concentraciones mayores, siendo el $\mathrm{N}$ el único que estuvo dentro de los rangos reportados por Hochmuth (1997). Por otro lado, García-Hernández et al. (2004) en chile güero, determinaron concentraciones promedio óptimas de $4.52,0.32,4.20,2.71$ y $0.67 \%$ de N, P, K, Ca y $\mathrm{Mg}$, respectivamente. Dichas concentraciones son similares a las encontradas, lo que permite destacar que las plantas de chile jalapeño, tienden a extraer mayores cantidades de $\mathrm{Ca}$ y $\mathrm{Mg}$ que las óptimas.

En el género Capsicum, planta C3, el orden decreciente de las concentraciones óptimas es $\mathrm{K}$ $>\mathrm{N}>\mathrm{Ca}>\mathrm{Mg}>\mathrm{P}$ (Tabla 2), lo que coincide con lo encontrado por Azofeifa y Moreira (2008) en tejido y planta completa de chile jalapeño. La misma tendencia fue obtenida por Arroyo-Vargas et al. (2013) en hojas de chile pimiento morrón (Tabla 3). Se ha reportado que el Ca controla la función de las acuaporinas, las cuales a su vez regulan la entrada de agua a través de las membranas biológicas y controlan la apertura estomática (Allen et al. 2001). El $80 \%$ de la función de distribución de varianza acumulada de DNC $r^{2}$ se asoció a un valor crítico de 3.1 (Figura 2), fue seleccionado para calificar a un grupo de plantas como la subpoblación de bajo rendimiento. Dicho valor crítico debe ser considerado en la validación de las normas de DNC, ya que cada base de datos independiente debe estar caracterizada por un valor similar (Khiari et al. 2001).

\section{Correlaciones entre nutrimentos y rendimiento} Las interacciones positivas o sinergismos del $\mathrm{P}$ con $\mathrm{K}$, y $\mathrm{Ca}$ con $\mathrm{Mg}$ fueron consistentes, estas interacciones se han reportado en sábila (GarcíaHernández et al. 2006) y para cultivos de chile correlaciones positivas entre N y P (GarcíaHernández et al. 2004). Se sabe que cada uno de los elementos tiene una función esencial en los procesos bioquímicos de la planta y por lo tanto con la productividad. Al respecto, Córdova-Sánchez et al. (2013) indican que el $\mathrm{N}$ es uno de los nutrientes más importantes para las plantas, ya que forma parte fundamental de las proteínas, aminoácidos y clorofila, por lo que es de los que más influyen en la obtención de altos rendimientos. La asociación positiva entre el $\mathrm{N}$ y $\mathrm{P}$ es la tendencia más sorprendente y consistente de forma estadística al considerar la diversidad de grupos taxonómicos y especies. Al 
analizar las tres matrices de correlaciones se determinó que el fósforo fue el nutrimento que más favoreció el rendimiento en el cultivar de chile Autlán. Al respecto Arroyo-Vargas et al. (2013) reportaron en chile pimiento morrón correlaciones significativas entre el rendimiento y $\mathrm{P}$, y entre el rendimiento y el $\mathrm{N}$ en sentido negativo; lo que evidencia la importancia de estos dos macro nutrimentos en el cultivo de chile.

La única interacción positiva en las tres matrices de correlaciones fue entre el $\mathrm{Ca}$ y $\mathrm{Mg}$, lo cual sugiere el sinergismo entre estos dos nutrimentos para el chile jalapeño y refuerza lo encontrado en chile güero y sábila (García-Hernández et al. 2004, 2006). Dentro de las interacciones negativas, la que se presentó en todos los casos fue la interacción $\mathrm{R}$ con la mayoría de los nutrimentos, lo cual es lógico y sigue la misma tendencia que lo encontrado por Arroyo-Vargas et al. (2013), ya que al aumentar las concentraciones de los nutrimentos, disminuye la concentración de los nutrientes no analizados denominados por $\mathrm{R}$ en el caso de DNC.

\section{CONCLUSIONES}

Las normas preliminares del diagnóstico de nutrientes, tomando como referencia un rendimiento mayor a $52.09 \mathrm{t} \mathrm{ha}^{-1}$, se asocian al siguiente porcentaje de composición foliar óptima de $\mathrm{N}(4.57 \pm 0.25), \mathrm{P}(0.18 \pm 0.03), \mathrm{K}(6.80 \pm$ 1.04), Ca $(2.46 \pm 0.17)$ y $\mathrm{Mg}(0.64 \pm 0.07)$. El orden decreciente de extracción de nutrientes en la floración fue $\mathrm{K}>\mathrm{N}>\mathrm{Ca}>\mathrm{Mg}>\mathrm{P}$, patrón similar al de otros cultivares de chile. Con respecto a las correlaciones de los nutrimentos, el fósforo fue el nutriente que más favoreció la obtención de altos rendimientos, lo que coincide con la significancia encontrada entre el $\mathrm{P}$ y $\mathrm{N}$ con el rendimiento. La única interacción positiva en las tres matrices de correlaciones fue entre el $\mathrm{Ca}$ y $\mathrm{Mg}$, lo cual sugiere el sinergismo entre estos dos nutrimentos para el cultivo chile. Es necesario continuar con estudios que validen las interacciones de nutrimentos en el cultivo de chile, para conocer los sinergismos y antagonismos al momento de aplicar una dosis de fertilización.

\section{AGRADECIMIENTOS}

Al Consejo Nacional de Ciencia y Tecnología (CONACYT) por la beca otorgada al primer autor. Al apoyo financiero por medio del proyecto SEP-CONACYT-CB 257808. Se agradece la colaboración del MC. Baudilio Acosta Vargas e Ing. Griselda Peña Armenta del laboratorio de espectrofotometría de absorción atómica del CIBNOR.

\section{LITERATURA CITADA}

Allen GJ, Chu SP, Harrington CL, Schumacher K, Hoffmann T; Tang YY, et al. (2001) A defined range of guard cell calcium oscillation parameters encodes stomatal movements. Nature 411: 1053-1057.

AOAC (2005) Official Methods of Analysis. 18th Edition. Association of Official Analytical Chemists. Washington, DC. 1108p.

Arroyo-Vargas L, Tirado-Torres JL, Volke-Haller VH, Valdez-Cepeda RD (2013) Normas preliminares de diagnóstico de nutrimento compuesto y correlaciones entre nutrimentos y rendimiento en pimiento (Capsicum annuum L.). Tropical and Subtropical Agroecosystems 16: 69-82.

Azofeifa A y Moreira MA (2008) Absorción y distribución de nutrimentos en plantas de chile jalapeño (Capsicum annuum L. cv. hot) en Alajuela, Costa Rica. Agronomía Costarricense 32: 19-29.

Bates TE (1971) Factors affecting critical nutrient concentrations in plants and their evaluation: a review. Soil Science 112: 116-130.

Blanco-Macías F, Lara-Herrera A, Valdez-Cepeda RD, Cortés-Bañuelos JO, Luna-Flores M, Salas-Luévano MA (2006) Interacciones nutrimentales y normas de la técnica de nutrimento compuesto en nopal 
(Opuntia ficus-indica L. Miller). Revista Chapingo Serie Horticultura 12: 165-175.

Bortolotti M (2013) Red pepper: from the kitchen to the pharmacy. Journal of Gastrointestinal and Liver Diseases 22: 253-256.

Bosland PW (2010) An American in Spain. In: Prohens J, Rodríguez-Burruezo A (ed). Advances in genetics and breeding of Capsicum and eggplant. Valencia, Spain. pp : 21-29.

Córdova-Sánchez S, Castelán-Estrada M, Cárdenas-Navarro R, Lobbit-Phellipe C, Peña-Cabriales JJ, VeraNúñez JA, et al. (2013) Fijación biológica de nitrógeno por cuatro fabáceas en suelos ácidos de Tabasco, México. Revista de la Facultad de Ciencias Agrarias de la Universidad Nacional de Cuyo 45: 1-9.

Delgado JA, Groffman PM, Nearing MA, Goddard T, Reicosky D, Lal R, et al. (2011) Conservation practices to mitigate and adapt to climate change. Journal of Soil and Water Conservation 66: 118-129.

FAOSTAT (2016) Food and Agriculture Organization of the United Nations. Modulo producción. http:// faostat.fao.org/site/339/default.aspx. Fecha de consulta 19 de febrero de 2016.

García-Hernández JL, Orona-Castillo I, González G, Valdez-Cepeda RD, Murillo-Amador B, Troyo-Diéguez $\mathrm{E}$, et al. (2009) Nutrient interactions and compositional nutrient diagnosis norms in pecan tree (Carya illinoensis). Revista Chapingo Serie Horticultura 15: 141-147.

García-Hernández JL, Valdez-Cepeda RD, Murillo-Amador B, Beltrán-Morales LF, Ruiz F, Orona-Castillo I, et al. (2006) Preliminary compositional nutrient diagnosis norms in Aloe vera L. grown on calcareous soil in an arid environment. Environmental and Experimental Botany 58: 244-252.

González-Zamora A, Sierra E, Luna JG, Pérez R, Rodríguez JC, García-Hernández JL (2013) Characterization of different Capsicum varieties by evaluation of their capsaicinoids content by high performance liquid chromatography, determination of pungency and effect of high temperature. Molecules 18: 1347113486.

González-Zamora A, Sierra E, Pérez R, Vázquez C, Gallegos MA, López JD, García-Hernández JL (2015) Measurement of capsaicinoids in chiltepin hot pepper: a comparison study between spectrophotometric method and high performance liquid chromatography analysis. Journal of Chemistry 2015: 1-10.

García-Hernández JL, Valdez-Cepeda RD, Murillo-Amador B, Nieto GA, Beltrán-Morales LF, MagallanesQuintanar R et al. (2004) Compositional nutrient diagnosis and main nutrient interactions in yellow pepper grown on desert calcareous soils. Journal of Plant Nutrition and Soil Science 167: 509-515.

Hanton EA (1992) Determination of potassium, calcium, and magnesium in plants by atomic absorption Techniques. In: Plank CO (Ed.), Plant analysis reference procedures for the southern region of the United States. Southern Cooperative series bulletin 368. University of Georgia, Athens. pp: 33-36.

Hochmuth GJ (1997) Pepper production guide for Florida. Commercial vegetable guide series. University of Florida, Cooperative Extension Service, Institute of Food and Agricultural Sciences, Gainesville, FL, USA. 4p.

Johnson PG, Koenig RT, Kopp KL (2003) Nitrogen, phosphorus, and potassium responses and requirements in calcareous sand greens. Agronomy Journal 95: 697-702.

Khiari L, Parent LE, Tremblay N (2001) Selecting the high-yield subpopulation for diagnosing nutrient imbalance in crops. Agronomy Journal 93: 802-808.

Macías-Duarte R, Grijalva CRL, Robles CF (2012) Respuesta de la aplicación de estiércol y fertilizantes sobre el rendimiento y calidad del chile jalapeño. Biotecnia 16: 32-38. 
Magallanes-Quintanar R, Valdez-Cepeda RD, Blanco-Macías F, Márquez M, Ruíz RR, Pérez O, et al. (2004) Compositional nutrient diagnosis in nopal (Opuntia ficus-indica). Journal of Professional Association for Cactus Development 6: 78-89.

Mateos RM, Jiménez A, Román P, Romojaro F, Bacarizo S, Leterrier M, et al. (2013) Antioxidant systems from pepper (Capsicum annuum L.): Involvement in the response to temperature changes in ripe fruits. International Journal of Molecular Sciences 14: 9556-9580.

Naiman A, Rosenfeld R, Zirkel G (1987) Introducción a la estadística. Editorial Mc Graw Hill. México, DF. 402p.

Nelson LA, Anderson RL (1977) Partitioning of soil test-crop response probability. In: Stelly M (ed.). Soil testing: Correlating and interpreting the analytical results. ASA Spec. Publ. 29. ASA, Madison, WI. pp: $19-38$.

Parent LE, Dafir M (1992) A theoretical concept of compositional nutrient diagnosis. Journal of the American Society for Horticultural Science 117: 239-242.

Valdez-Cepeda RD, Magallanes-Quintanar R, Blanco-Macías F, Hernández-Caraballo EA, García-Hernández JL (2013) Comparison among boltzmann and cubic polynomial models for estimation of compositional nutrient diagnosis dtandards: Opuntia ficus-indica L. case. Journal of Plant Nutrition 36: 895-910.

Vera-Guzmán AM, Chávez-Servia JL, Carrillo-Rodríguez JC, López MG (2011) Phytochemical evaluation of wild and cultivated pepper (Capsicum annuum L. and C. pubescens Ruiz \& Pav .) from Oaxaca, México. Chilean Journal of Agricultural Research 71: 578-585.

Walworth JL, Sumner ME (1987) The diagnosis and recommendation integrated system (DRIS). Advances in Soil Science 6: 149-188.

Ware GO, Ohki K, Moon LC (1982) The mitscherlich plant growth model for determining critical nutrient deficiency levels. Agronomy Journal 74: 88-91. 